\title{
Lot6p from Saccharomyces cerevisiae is a FMN-dependent reductase with a potential role in quinone detoxification
}

\author{
Sonja Sollner ${ }^{1}$, Ruth Nebauer ${ }^{1}$, Heidemarie Ehammer ${ }^{1}$, Anna Prem ${ }^{1}$, Sigrid Deller ${ }^{1}$, \\ Bruce A. Palfey ${ }^{2}$, Günther Daum ${ }^{1}$ and Peter Macheroux ${ }^{1}$ \\ 1 Graz University of Technology, Institute of Biochemistry, Graz, Austria \\ 2 Department of Biological Chemistry, University of Michigan, Ann Arbor, MI, USA
}

\section{Keywords \\ DT-diaphorase; Lot6p; NQO1; quinone \\ reductase; redox cycling \\ Correspondence \\ P. Macheroux, Institute of Biochemistry, Graz University of Technology, Petersgasse 12/II, A-8010 Graz, Austria \\ Fax: +43 3168736952 \\ Tel: +433168736450 \\ E-mail: peter.macheroux@tugraz.at}

(Received 14 November 2006, revised 18 December 2006, accepted 8 January 2007)

doi:10.1111/j.1742-4658.2007.05682.x
NAD(P)H:quinone acceptor oxidoreductases are flavoenzymes expressed in the cytoplasm of many tissues and afford protection against the cytotoxic effects of electrophilic quinones by catalyzing a strict two-electron reduction. Such enzymes have been reported from several mammalian sources, e.g. human, mouse and rat, and from plant species. Here, we report identification of Lot6p (YLR011wp), the first soluble quinone reductase from the unicellular model organism Saccharomyces cerevisiae. Localization studies using an antibody raised against Lot6p as well as microscopic inspection of Lot6p-GFP demonstrated accumulation of the enzyme in the cytosol of yeast cells. Despite sharing only $23 \%$ similarity to type 1 human quinone reductase, Lot6p possesses biochemical properties that are similar to its human counterpart. The enzyme catalyzes a two-electron reduction of a series of natural and artificial quinone substrates at the expense of either NADH or NADPH. The kinetic mechanism follows a ping-pong bi-bi reaction scheme, with $K_{\mathrm{M}}$ values of 1.6-11 $\mu \mathrm{M}$ for various quinones. Dicoumarol and Cibacron Marine, two well-known inhibitors of the quinone reductase family, bind to Lot6p and inhibit its activity. In vivo experiments demonstrate that the enzymatic activity of Lot6p is consistent with the phenotype of both $\Delta$ lot6 and Lot6p overexpressing strains, suggesting that Lot6p may play a role in managing oxidative stress in yeast.
Several cellular oxidoreductases are involved in the metabolism of quinone compounds by catalyzing oneor two electron reductions of quinones. The cytotoxic effects of quinones are commonly thought to arise from their one-electron reduction, for example, by cytochrome $\mathrm{P} 450$ reductases, resulting in oxidative cycling of deleterious oxygen species.

In contrast to other cellular reductases, NAD(P)H:quinone acceptor oxidoreductases (QRs) catalyze strict two-electron reductions and thereby afford protection against cytotoxic and neoplastic effects of electrophilic quinones [1]. For example, it has been shown that QR-null mice are more susceptible to quinone toxicity than their wild-type counter- parts [2]. Furthermore, QRs are found in many solid tumors at increased levels and therefore can be used to target the tumor cells through bioreductive activation of quinone-based pro-drugs such as mitomycins [3].

Cytosolic mammalian QRs have been crystallized from human (quinone reductase type 1 and 2) [4,5], rat [6] and mouse [4]. All of them are homodimers, containing FAD as a cofactor and exhibiting a flavodoxin-like fold that is characterized by twisted, parallel $\beta$-strands at the centre flanked by helices on both sides. The FAD cofactor is bound to the enzyme in the same manner, with the isoalloxazine ring system of the molecule accessible to the solvent and the ribityl phosphate and AMP moiety buried in the protein.

\footnotetext{
Abbreviations

duroquinone, 2,3,5,6-tetramethyl-1,4-benzoquinone; GFP, green fluorescent protein; Ni-NTA, nickel-nitrilotriacetic acid agarose; QR, quinone reductase.
} 
Additionally, several soluble QRs were reported in bacteria and fungi, including the QRs from Escherichia coli [7] and the basidiomycete Phanerochaete chrysosporium [8] The QR from E. coli was shown to adopt a structure similar to horse liver alcohol dehydrogenase and lacks a flavin cofactor, and therefore is structurally not related to the mammalian QRs. In contrast to that, the P. chrysosporium $\mathrm{QR}$ is a FMN-containing enzyme, capable of reducing a wide range of quinone substrates and ferricyanide [8]. The latter enzyme shows significant sequence similarity to WrbA from E. coli, a multimeric flavodoxin, and hence may display a similar structural topology as mammalian QRs.

Many QRs were found to act via a ping-pong bi-bi mechanism, whereby the oxidized enzyme is firstly reduced by $\mathrm{NAD}(\mathrm{P}) \mathrm{H}$ (or, in the case of human $\mathrm{QR}$ type 2, nonphosphorylated nicotinamide derivatives) and subsequently the reduced enzyme intermediate reduces the quinone acceptor. Thus, the role of $\mathrm{QR}$ in animal cells is assumed to be the detoxification of redox-active compounds (such as quinones) produced by the metabolism of carcinogenic aromatic hydrocarbons $[9,10]$. Therefore, human QR expression was shown to be induced by a variety of redox active compounds [1], and both an antioxidant response element [11] and a xenobiotic response element have been found in the promoter region of human QR [12].

In the yeast Saccharomyces cerevisiae, five genes were identified whose expression is up-regulated in response to low temperature [13]. Elucidation of the X-ray crystal structure of one of the LOT ( $L O \mathrm{w} T \mathrm{em}$ perature Responsive) gene products, termed Lot6p, revealed a flavodoxin-like fold, self-association as a homodimer and the presence of one FMN cofactor per subunit [14]. Due to its close structural homology to Bacillus subtilis YhdA that recently was reported to reductively cleave the $-\mathrm{N}=\mathrm{N}$ - bonds in azo dyes [15], Lot6p was annotated as an azoreductase. Lot6p reductase activity was characterized by testing with several azo dyes, but also with nitro compounds and ferricyanide as electron acceptors. It was shown that both NADH and NADPH can act as an electron donor, with NADPH being more efficient. Ferricyanide was reported to be the best substrate for reduction; only very weak azoreductase and nitroreductase activities could be detected [14]. Thus, no definite catalytic function could be ascribed to Lot $6 \mathrm{p}$ from the structure up to now.

In our work, we analyzed Lot6p for its ability to act as a quinone reductase by testing several artificial as well as naturally occurring quinones as substrates. Here, we report that Lot6p exhibits high activity towards a variety of quinone substrates by reducing them via a ping-pong bi-bi mechanism, and that quinone reductase activity requires the binding of the quinone substrate to the active site. This type of activity has not been reported before in S. cerevisiae.

In an effort to examine the physiological role of Lot6p, we assessed the relevance of this enzyme in oxidative stress response by comparing the viability of wild type, Lot6p knock-out and Lot6p overexpressing yeast strains following the administration of an oxidative stressor. The results from these experiments indicate that Lot6p may play a role in protecting yeast cells against quinone and semiquinone toxicity and their potential for the generation of reactive oxygen species.

\section{Results}

\section{Subcellular localization of Lot6p}

As a prerequisite for isolation of Lot6p from the yeast $S$. cerevisiae we investigated the subcellular localization of this enzyme. Ambiguous reports in the literature $[31,32]$ describing occurrence of this polypeptide in the cytosol on the one hand and in the nucleus on the other hand were the reason for this re-investigation. Western blot analysis using a primary antibody against Lot6p (Fig. 1A) revealed that Lot6p was enriched in the cytosolic fraction. Weak signals were also detected in the microsomal fractions and in the vacuoles, which were due to cross-contamination of these fractions with cytosol as determined in a control using an antibody against the cytosolic marker protein glyceraldehyde-3-phosphate dehydrogenase (data not shown).

Additionally, an N-terminal GFP-Lot6p hybrid was constructed. Fluorescence microscopic inspection demonstrated that this polypeptide was mainly localized to the cytosolic fraction with a certain amount of the protein being present in the nucleus (Fig. 1B) confirming data previously provided in genome-wide studies $[31,32]$. The apparent discrepancy between fluorescence microscopic data and western blot analysis may be explained by the fact that nuclei have a tendency to lose their soluble content during the cell fractionation procedure.

\section{Isolation of Lot6p-His6}

Cloning of the LOT6 gene into the isopropyl-1-thioD-galactopyranoside inducible expression vector pET21a and transformation of E. coli Rosetta(DE3)pLysS cells leads to the expression of Lot6p-His6 that amounts to a sizable fraction of the total soluble protein (Fig. 2, insert). As Lot6p-His6 is expressed as 


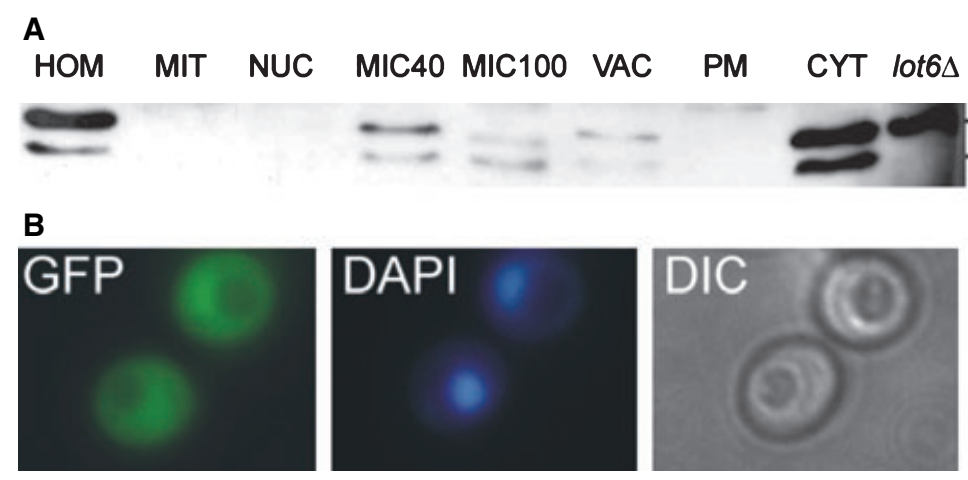

Fig. 1. Localization of GFP-Lot6p by western blot analysis and fluorescence microscopy. (A) Western blot analysis of organelles from S. cerevisiae wild-type BY4741. Proteins $(20 \mu \mathrm{g})$ from subcellular fractions were immunoblotted with antibodies against Lot6p. HOM, cell homogenate; MIT, mitochondria; NUC, nuclei; MIC40, $40000 \mathrm{~g}$ microsomes; MIC100, $100000 \mathrm{~g}$ microsomes; VAC, vacuoles; PM, plasma membrane; CYT, cytosol; *, background band. As a control, cell homogenate from a lot64 strain prepared by alkaline lysis [45] is shown. (B) GFP fluorescence of Lot6p-GFP and staining of DNA with DAPI. Details of the method are described in the Methods section. As a control, differential interference contrast (DIC) images are shown.

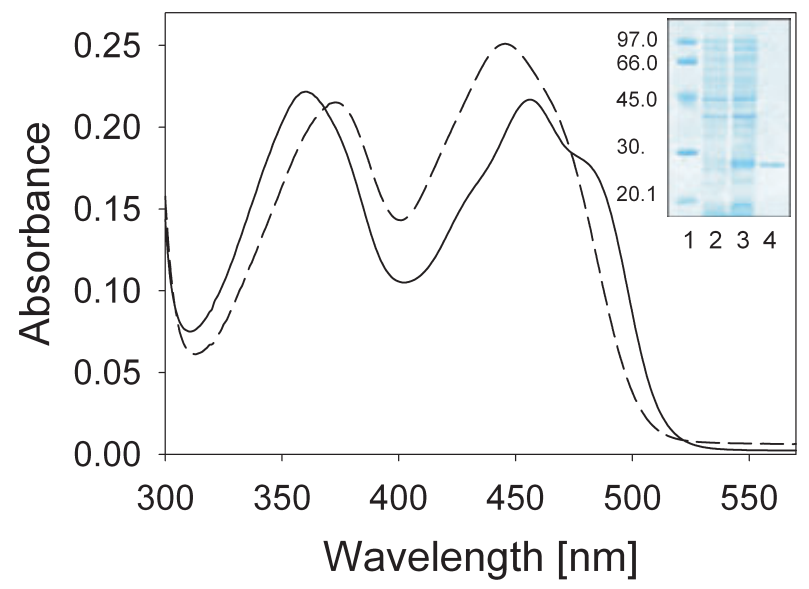

Fig. 2. UV-visible absorbance spectra of Lot6p before and after denaturation. Solid and dashed lines represent the spectrum of Lot6p and free FMN cofactor, respectively. Denaturation of purified Lot6p was carried out in storage buffer (20 mM Tris/HCl, pH 8.0) containing $0.2 \%$ SDS. The insert shows SDS/PAGE of Lot6p expression and purification. Lane 1, molecular mass standard as indicated; lane 2, protein extract before induction of Lot6p; lane 3, protein extract after induction of Lot6p; lane 4, purified protein Lot6p. Proteins were visualized by Coomassie blue staining.

a C-terminally hexahistidine-tagged protein, it could be purified to apparent homogeneity by a one-step nickel-nitrilotriacetic acid agarose (Ni-NTA) affinity chromatography yielding about $250 \mathrm{mg}$ of purified polypeptide from $15 \mathrm{~g}$ of wet biomass. The UV-vis absorbance spectrum of Lot6p-His6 shows two distinct peaks at 360 and $455 \mathrm{~nm}$, with a shoulder at $480 \mathrm{~nm}$, clearly demonstrating the presence of a flavin cofactor (Fig. 2). This finding is in keeping with the threedimensional structure of the enzyme (pdb entry 1t0i), showing a noncovalently attached FMN molecule per subunit [14]. Denaturation of the protein, and therefore release of the cofactor, produced a hypsochromic shift of the absorbance maximum at $455 \mathrm{~nm}$ and a bathochromic shift of the maximum at $360 \mathrm{~nm}$, being characteristic for the release of free FMN. Using an extinction coefficient of $\varepsilon_{450}=12400 \mathrm{M}^{-1} \cdot \mathrm{cm}^{-1}$ for free FMN, the extinction coefficient for Lot6p-His6-bound FMN was determined to $\varepsilon_{455}=10690 \mathrm{M}^{-1} \cdot \mathrm{cm}^{-1}$.

\section{Biochemical properties of Lot6p}

In order to further characterize the properties of Lot6p-His6, the enzyme was subjected to gradual photoreduction under anoxic conditions. The flavin is continuously reduced with an isosbestic point at $320 \mathrm{~nm}$. Under these conditions, a flavin semiquinone was not observed. Upon reoxidation with air, the initial absorbance spectrum of the flavin cofactor was restored (Fig. 3). To measure the potential of the FMN/FMNH ${ }_{2}$ couple in Lot6p, a reductive titration was carried out in the presence of 1-hydroxyphenazine $\left(E_{\mathrm{m}}\right.$ at $\mathrm{pH} 7.0=-0.172 \mathrm{~V}$ [33]), according to the method of Massey [30]. The amounts of oxidized and reduced indicator dye as well as the amount of oxidized and reduced Lot6p were quantitated at 320 and $342 \mathrm{~nm}$, respectively. The $\log (\mathrm{ox} / \mathrm{red})$ for the 1-hydroxyphenazine was plotted versus the $\log$ (ox/red) for the enzyme-bound FMN (Fig. 4, inset), according to the method of Minneart [34]. A 1-unit slope was calculated, as expected for equilibrium between a two-electron acceptor dye and a two-electron donor without semiquinone formation, giving a value of $-172 \mathrm{mV}$ for the $\mathrm{FMN} / \mathrm{FMNH}_{2}$ potential. 


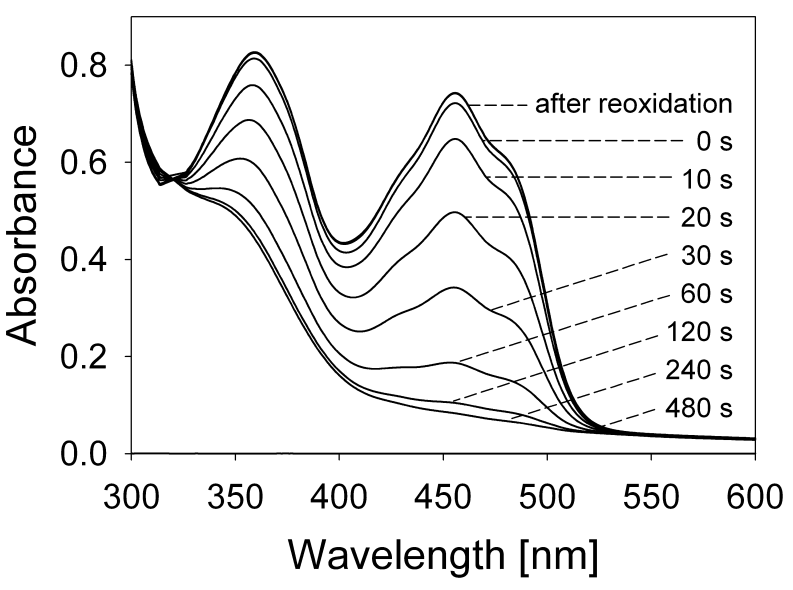

Fig. 3. Anaerobic photoreduction of Lot6p. Lot6p $(60 \mu \mathrm{m})$ in $20 \mathrm{~mm}$ Tris $/ \mathrm{HCl}$ buffer, $\mathrm{pH} 8.0$, containing $1 \mathrm{mM}$ EDTA was made anaerobic by repeated cycles of evacuation and flushing with oxygenfree nitrogen gas. The second spectrum from top was recorded after anaerobic conditions were established. The sample was then photoreduced using a 50-W halogen lamp as a light source. Spectra shown were recorded after 10,20,30,60, 120, 240 and $480 \mathrm{~s}$ (from top to bottom). The cuvette was then opened to air and the spectrum of the reoxidized sample was recorded.

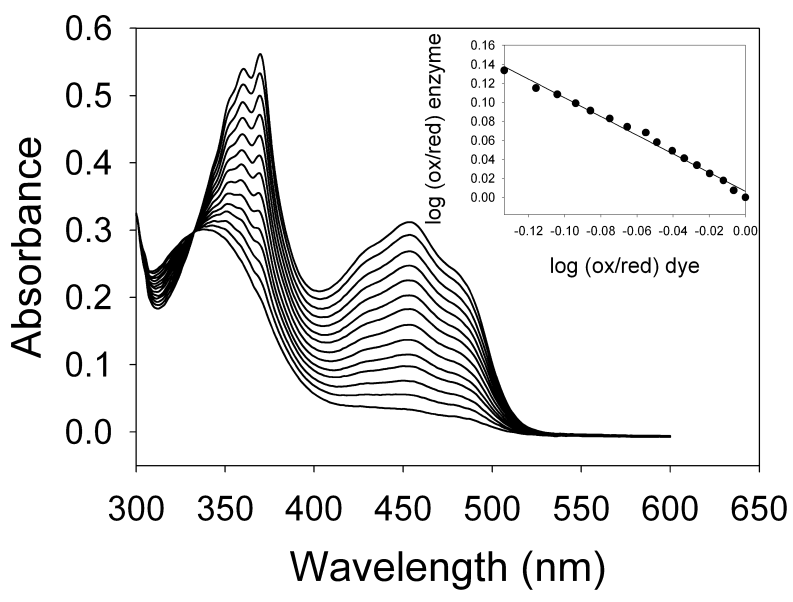

Fig. 4. Anaerobic determination of Lot6p redox potential by equilibration with a redox dye. Lot $6 \mathrm{p}, 30 \mu \mathrm{m}$ in $50 \mathrm{~mm}$ phosphate buffer, $\mathrm{pH} 7.0,25.0^{\circ} \mathrm{C}$, containing $2 \mu \mathrm{M}$ methyl viologen and about $25 \mu \mathrm{M}$ 1-hydroxyphenazine was titrated with dithionite $\left(1 \mathrm{mg} \cdot \mathrm{mL}^{-1}\right.$ in $50 \mathrm{~mm}$ phosphate, $\mathrm{pH}$ 7.0) under anaerobic conditions. Inset, data obtained while monitoring reduction of hydroxyphenazine at $320 \mathrm{~nm}$ and reduction of enzyme-bound FMN at $342 \mathrm{~nm}$.

This potential is $35 \mathrm{mV}$ more positive than that of free flavin $(-207 \mathrm{mV}$ at $\mathrm{pH} 7.0)$ and corresponds to the midpoint potential found for human QR type 1 $(-159 \mathrm{mV}[39])$.

Suzuki et al. [35] reported that a homologous enzyme from Bacillus sp. OY1-2, which has $32 \%$

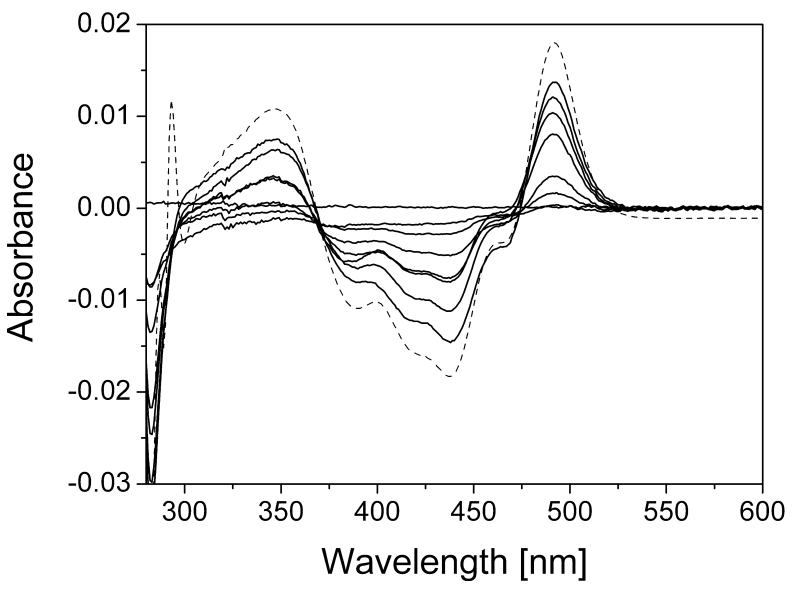

Fig. 5. Titration of apoLot6p with FMN. ApoLot6p (20 $\mu \mathrm{m})$ in $20 \mathrm{~mm}$ Tris/ $\mathrm{HCl}$ buffer, $\mathrm{pH} 8.0$, was prepared as described in the Experimental procedures. Titration experiment; for clarity, representative spectra at $0,2.2,4.4,8.8,17.5,30.2,62.5$ and $96.4 \mu \mathrm{M}$ FMN concentration are depicted (from top to bottom at $440 \mathrm{~nm}$ ). The difference spectrum of native and denatured Lot6p is shown as a dashed line.

sequence identity with Lot6p, lacks a flavin cofactor. The apparent absence of a flavin cofactor from this enzyme is difficult to reconcile with the available X-ray crystal structures of both Lot6p and YhdA from B. subtilis, an enzyme that has $53 \%$ sequence identity with the protein from Bacillus sp. OY1-2. Both X-ray crystal structures clearly demonstrate the presence of FMN in the active site [14]. Therefore, we subjected Lot6p-His6 to a HiTrap Blue affinity column which is very similar to the Red-Sepharose CL-6B column that was used by Suzuki et al. [35] for protein purification. During washing of the column with phosphate buffer, free FMN cofactor was eluted. Subsequently, the protein was eluted with $1.5 \mathrm{M} \mathrm{KCl}$, with no indication of any remaining flavin cofactor. In order to determine whether this process is reversible, the subsequent binding of FMN to apoLot6p-His6 was monitored by difference UV-visible spectroscopy, the results of which are shown in Fig. 5. The observed spectral changes are characterized by a hyperchromic effect on flavin absorbance peaking at 350 and $490 \mathrm{~nm}$ and a hypochromic effect peaking at $445 \mathrm{~nm}$ with concurrent isosbestic points at 370 and $475 \mathrm{~nm}$. The resulting spectrum is very similar to the difference spectrum of native and denatured Lot6p-His6 (Fig. 5, dashed line), suggesting complete reversibility of cofactor removal, which was also shown for the QR from P. chrysosporium [8]. Therefore, we conclude that FMN-depletion can occur during protein purification leading to erroneous assumptions concerning cofactor requirements for this class of enzymes. 


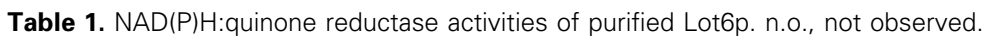

\begin{tabular}{|c|c|c|c|c|}
\hline Substrate & $K_{\mathrm{M}}(\mu \mathrm{M})$ & $k_{\text {cat }}\left(s^{-1}\right)$ & $k_{\mathrm{cat}} / K_{\mathrm{M}}\left(\mathrm{M}^{-1} \cdot \mathrm{s}^{-1}\right)$ & $K_{\mathrm{I}}(\mu \mathrm{M})$ \\
\hline 1,4-Benzoquinone & $2.7 \pm 0.4$ & $21.1 \pm 0.9$ & $7.7 \times 10^{6}$ & n.o. \\
\hline Coenzyme $\mathrm{Q}_{0}$ & $1.6 \pm 0.2$ & $13.3 \pm 0.5$ & $8.1 \times 10^{6}$ & n.o. \\
\hline Duroquinone & $11.0 \pm 3.1$ & $6.3 \pm 0.5$ & $5.7 \times 10^{5}$ & n.o. \\
\hline 1,4-Naphthoquinone & $1.5 \pm 0.2$ & $16.0 \pm 0.5$ & $1.1 \times 10^{7}$ & $188 \pm 32$ \\
\hline 1,4-Anthraquinone & n.o. & n.o. & - & n.o. \\
\hline Coenzyme $\mathrm{Q}_{10}$ & n.o. & n.o. & - & n.o. \\
\hline
\end{tabular}

\section{Enzymatic properties of Lot6p}

The structural similarity between mammalian QRs and Lot6p led us to examine the ability of Lot6p to catalyze the reduction of quinones. The reduction process was monitored by following the loss of absorbance of $\mathrm{NADPH}$ rather than NADH, as suggested by Liger et al. [14], that acts as the electron donor. Several naturally occurring as well as artificial quinones were tested as electron acceptors. A summary of the obtained kinetic parameters is given in Table 1 . The enzyme can use 1,4-benzoquinone, duroquinone, 1,4-naphthoquinone and coenzyme $\mathrm{Q}_{0}$ as electron acceptor. The velocity of quinone reduction shows an inverse correlation with hydrophobicity, as previously shown for the sugarbeet enzyme [36]. Although 1,4-benzoquinone showed the highest turnover number, 1,4-naphthoquinone was found to be the best acceptor on the basis of the $k_{\text {cat }}: K_{\mathrm{M}}$ ratios. Relatively lipophilic quinones such as 1,4-anthraquinone and coenzyme $\mathrm{Q}_{10}$ were not reduced at all. Substrate inhibition was observed for 1,4-naphthoquinone, as previously reported for human QR type 1 with 9,10-anthraquinone as the substrate [37].

The reaction mechanism of Lot6p has been investigated by steady-state kinetic analysis. Initial velocity measurements performed in the presence of NADPH as the electron donor and 1,4-benzoquinone as electron acceptor resulted in a family of parallel lines in a double-reciprocal plot, indicating a ping-pong bi-bi mechanism (Fig. 6). This finding is in agreement with previous observations for several other QRs, as those enzymes use a unique catalytic site for both co-substrate and substrate binding. More precisely, the electron donor (co-substrate, i.e. NADPH) occupies the FMN site firstly, and only after its release, can the electron-accepting species (substrate, i.e. quinone) enter the active site. This result is particularly intriguing, as the Lot6p homolog in B. subtilis, termed YhdA, was recently reported to form a ternary complex in a bi-bi ternary mechanism, i.e. both $\mathrm{NAD}(\mathrm{P}) \mathrm{H}$ and the electron acceptor (FMN) must bind to the active site before catalysis can occur [15].

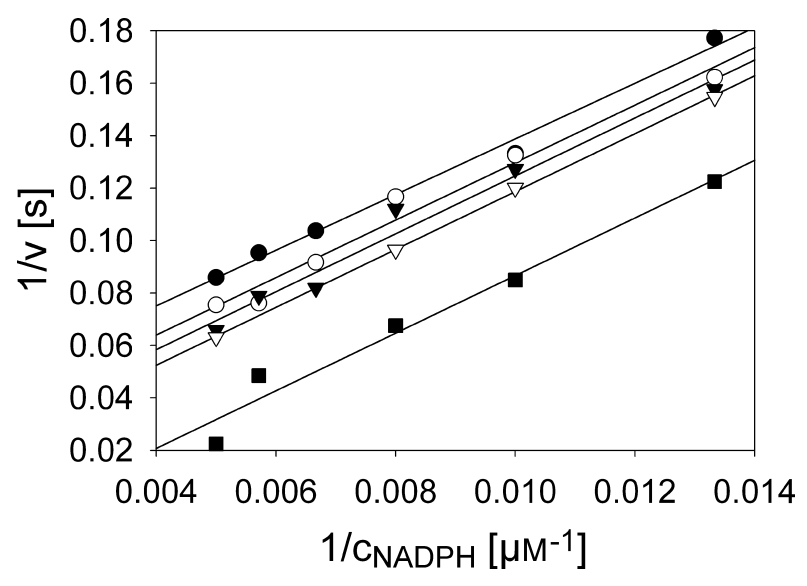

Fig. 6. Rate of $\mathrm{NADPH}$ oxidation as a function of 1,4-benzoquinone concentration. Double reciprocal plot of initial rates versus concentrations of NADPH (50-200 $\mu \mathrm{m})$ measured at $340 \mathrm{~nm}$. NADPH oxidation activity was assayed at $37^{\circ} \mathrm{C}$ at varying concentrations of 1,4-benzoquinone. Benzoquinone concentrations used were $2 \mu \mathrm{M}$ $(\bullet), 5 \mu \mathrm{M}(\bigcirc), 10 \mu \mathrm{M}(\mathbf{\nabla}), 15 \mu \mathrm{M}(\nabla)$, and $30 \mu \mathrm{M}$ (

In order to demonstrate binding of quinone substrates to the FMN site of Lot6p, we performed several titration experiments. Binding of quinones, which do not absorb in the same wavelength area as the flavin cofactor, was directly monitored by titrating Lot6p-His6 with quinone substrates (Fig. 7A, for the titration of Lot6p-His6 with coenzyme $\mathrm{Q}_{0}$ ). In the case that substrate absorbance interfered with flavin absorbance (e.g. 1,4-benzoquinone), binding was monitored by difference UV-visible absorbance spectrophotometry, the results of which are shown in Fig. 7B. Titration of Lot6p-His6 with 1,4-benzoquinone, duroquinone and 1,4-naphthoquinone showed saturation behavior, and a hyperbolic fit to the data produced dissociation constants in the range of 20-77 $\mu \mathrm{M}$ (Table 2). Although coenzyme $\mathrm{Q}_{0}$ was found to give rise to similar spectral changes as the substrates mentioned above, no saturation was found up to a concentration of $400 \mu \mathrm{M}$. Due to the poor aqueous solubility of coenzyme $\mathrm{Q}_{0}$, the maximal concentration of the substrate was limited to this range. 1,4-Anthraquinone and 


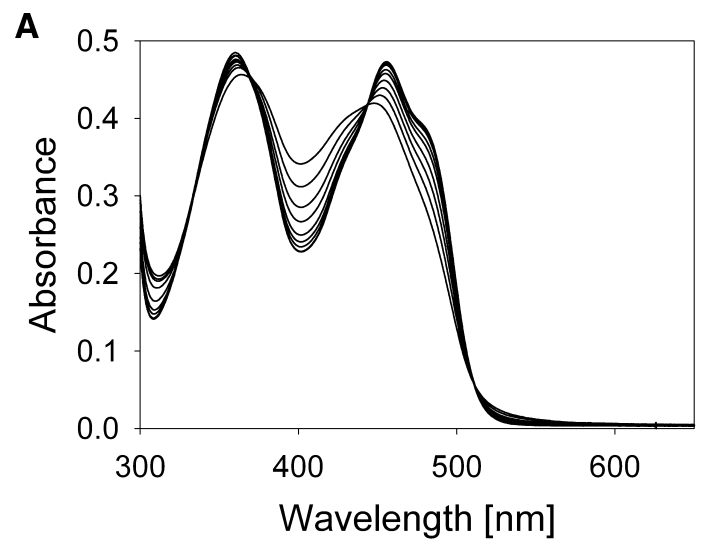

B

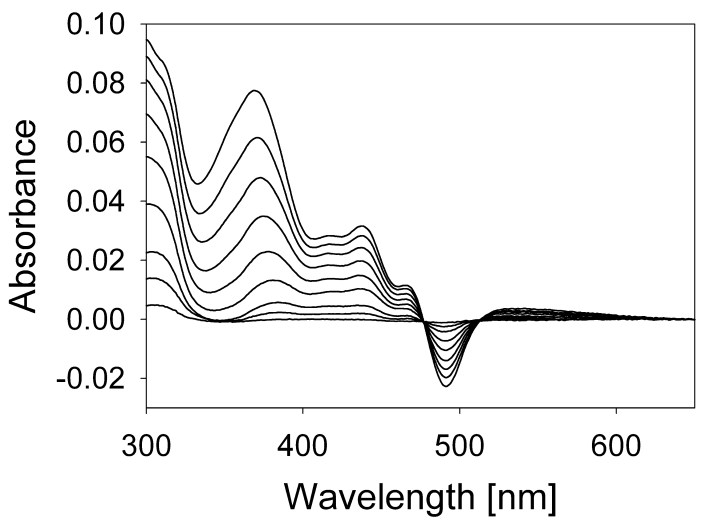

Fig. 7. Titration of Lot6p with quinone substrates. (A) Lot6p (50 $\mu \mathrm{m}$, in $50 \mathrm{~mm}$ phosphate buffer, $\mathrm{pH}$ 8.0) was titrated with coenzyme $\mathrm{Q}_{0}$. For clarity, representative spectra at $0,5.5,21.7,47.6,76.9$, $130,200,294$, and $410 \mu \mathrm{M}$ coenzyme $\mathrm{Q}_{0}$ concentration are depicted (from top to bottom at $460 \mathrm{~nm}$ ). (B) Lot6p (50 $\mu \mathrm{M}$, in $50 \mathrm{~mm}$ phosphate buffer, $\mathrm{pH} 8.0$ ) was titrated with 1,4-benzoquinone. For clarity, representative spectra at 0, 5.5, 16.4, 27.0, 47.6, 67.4, 95.4, 130, 163, 234 and $294 \mu \mathrm{M}$ 1,4-benzoquinone concentrations are depicted (from top to bottom at $490 \mathrm{~nm}$ ).

Table 2. Dissociation constants for several quinone substrates with Lot6p. Lot6p (10 $\mu \mathrm{M}$, in $50 \mathrm{~mm}$ phosphate buffer, $\mathrm{pH}$ 8.0) was titrated with various quinones $(c=5.5-294 \mu \mathrm{M})$. The results shown are means of three independent experiments. n.o., not observed.

\begin{tabular}{ll}
\hline Substrate & $K_{d}(\mu \mathrm{M})$ \\
\hline 1,4-Benzoquinone & $38.5 \pm 6.2^{\mathrm{a}}$ \\
Coenzyme $\mathrm{Q}_{0}$ & $>200^{\mathrm{a}, \mathrm{b}}$ \\
Duroquinone & $76.9 \pm 20.9^{\mathrm{a}, \mathrm{b}}$ \\
1,4-Naphthoquinone & $19.9 \pm 6.7^{\mathrm{a}, \mathrm{b}}$ \\
1,4-Anthraquinone & n.o. ${ }^{\mathrm{a}}$ \\
Coenzyme $\mathrm{Q}_{10}$ & n.o. ${ }^{a}$ \\
\hline
\end{tabular}

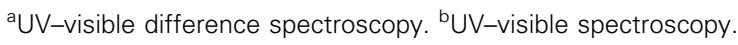

coenzyme $\mathrm{Q}_{10}$ did not bind to the FMN site of Lot6pHis6.

The QRs from human (type 1), mouse, rat, and $P$. chrysosporium were reported to be strongly inhibited by dicoumarol, a vitamin $\mathrm{K}$ antagonist, and hence we were interested to test the ability of dicoumarol to inhibit Lot6p. Dicoumarol in fact competitively inhibits quinone reductase activity of Lot6p-His6 with respect to NADPH. A replot of the slopes versus inhibitor concentrations yields a $K_{\mathrm{I}}$ value of $0.41 \mu \mathrm{M}$ (data not shown). The same inhibition pattern was found for Cibacron Marine, an azo dye, with a resulting $K_{\mathrm{I}}$ of $16 \mu \mathrm{M}$ (data not shown). Binding of dicoumarol to the active site was further verified by titrating Lot6p-His6 with inhibitor, giving rise to spectral changes, clearly suggesting binding of dicoumarol to the flavin site of Lot6p (data not shown).

\section{Influence of Lot6p on quinone toxicity}

With the aim of investigating the physiological role of Lot6p in S. cerevisiae, we characterized the phenotype of the wild type-, Lot6p overexpression- and Alot6 strain in oxidative stress resistance. If Lot6p in fact was responsible for detoxifying quinones, it is expected that yeast lacking Lot6p would be more susceptible to quinone toxicity. Conversely, a Lot6p overexpression strain would be more resistant towards quinones as compared with wild type.

To test this hypothesis, yeast cells were exposed to 1,4-naphthoquinone under aerobic conditions and the effect on growth was determined as a function of time. 1,4-Naphthoquinone was taken as a model compound for quinone toxicity, as Rodriguez et al. [29] showed evidence of redox cycling-mediated toxicity for this quinone. To ensure that the observed sensitivity to oxidative stress is completely attributed to the quinone rather than to dimethyl sulfoxide (the solvent), control cultures were grown in the presence of the same amount of dimethyl sulfoxide but lacking the quinone stressor.

The results extracted from theses growth curves are shown in Fig. 9 and are expressed as the percentage of viability relative to the control culture. Deletion of Lot6p increased the toxicity of 1,4-naphthoquinone considerably; only $53 \%$ of the cells were viable after $8 \mathrm{~h}$, compared with $70 \%$ of the wild-type cells. Correspondingly, yeast cells overexpressing Lot6p were less susceptible to quinone stress, resulting in $84 \%$ relative viability after $8 \mathrm{~h}$.

\section{Discussion}

Sequence analysis of Lot6p from S. cerevisiae led to the assignment of the protein to the NADPH-dependent FMN reductase family. In yeast, the amount of mRNA encoding for Lot6p has been found to be 
increased in response to low-temperature exposure [13].

Recently, Liger et al. [14] solved the crystal structure of Lot6p, revealing a tertiary and quaternary structure similar to structures of mammalian quinone reductases. X-ray structures of enzymes which belong to this family are available from human (QR type 1 and 2, pdb entry code $1 \mathrm{~d} 4 \mathrm{a}$ and $1 \mathrm{qr} 2$, respectively), mouse (pdb entry code 1dxq) and rat (pdb entry code 1qrd). All of them adopt a typical flavodoxin-like fold, consisting of a central five-stranded parallel $\beta$-sheet sandwiched by $\alpha$-helices. Despite the nature of the flavin cofactor, which is FMN for Lot6p instead of FAD in the case of the mammalian QRs, the proteins share a common overall topology (23\% sequence identity of human QR type 1 and Lot6p, rmsd of $10.4 \AA$ over 1318 atoms), as can be seen from Fig. 8. The flavin cofactor is bound in a similar manner, with the re-face buried and the si-face largely exposed to the solvent. Moreover, Lot6p features a putative nucleotide binding motif (GGHGG) at the edge of the pyrimidine ring moiety which may be used by $\mathrm{NAD}(\mathrm{P}) \mathrm{H}$ to dock in direct vicinity to the FMN cofactor. Such a binding was shown for mammalian QR type 1 and $\mathrm{NADP}^{+}$, where the nicotinamide ring stacks upon the si-face of the isoalloxazine ring [6].

For mammalian QRs, quinone substrates were shown to bind to the same site in order to sequester the electrons from the reduced flavin cofactor in a ping-pong bi-bi mechanism. In the case of Lot6p, we could clearly demonstrate that the enzyme also obeys an ordered ping-pong mechanism, whereby native oxidized Lot6p is first reduced by NADPH and subsequently the reduced enzyme intermediate is reoxidized by the quinone.

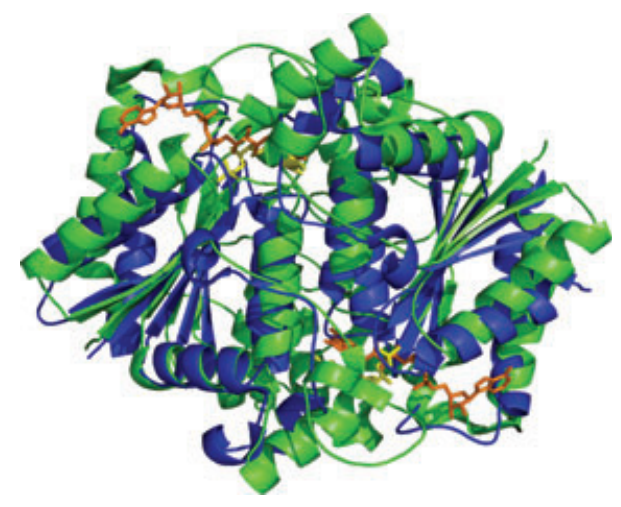

Fig. 8. Comparison of human $Q R$ type 1 and Lot6p. Ribbon representation of Lot6p from S. cerevisiae (shown in blue, pdb entry 1t0i) and human QR type 1 (shown in green, pdb entry 1d4a). The FMN cofactors of Lot6p are shown as stick models in yellow, FADs of $\mathrm{QR}$ are shown in orange.

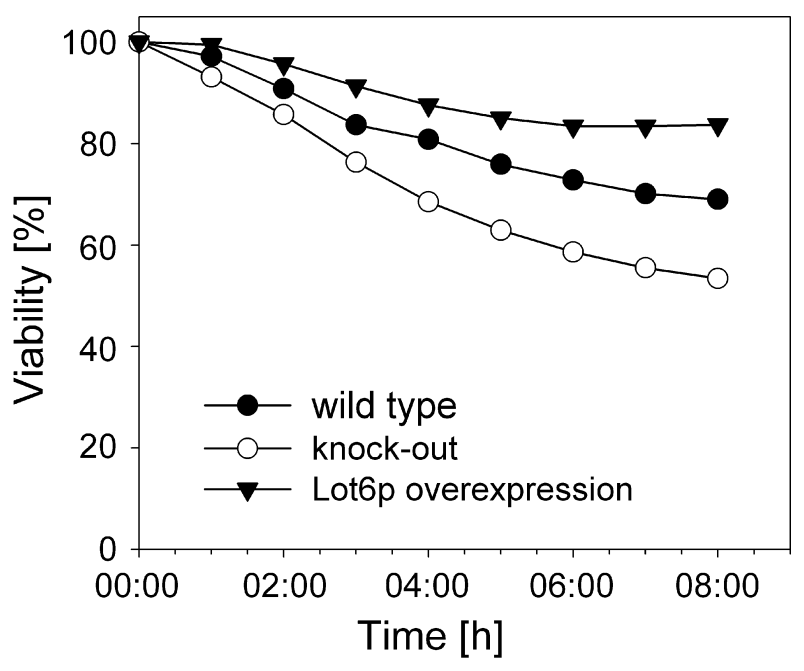

Fig. 9. Viability of yeast strains under quinone stress. The growth rate was monitored by hourly optical density measurements at $600 \mathrm{~nm}$. Values on $y$-axis represent the ratio to that of untreated cells (in per cent). The data points are means of four independent experiments.

To further confirm binding of quinones to the FMN site of Lot6p, we performed several titration experiments. Binding could be demonstrated for 1,4-benzoquinone, 1,4-naphthoquinone, duroquinone and coenzyme $\mathrm{Q}_{0}$. Interestingly, affinity of quinone substrates to the FMN site of Lot6p appears to correlate with their hydrophobicity, i.e. quinones which are more apolar show lower dissociation constants. This might be due to a relatively hydrophobic environment in the vicinity of the FMN cofactor which favors hydrophobic interactions between the active site residues and quinone substrates. However, quinones which are highly apolar but too bulky cannot enter the active site, as shown for 1,4-anthraquinone and coenzyme $\mathrm{Q}_{10}$. To shed more light on the precise binding mode of substrates to the active site, we are currently in the process of co-crystallizing Lot6p and several quinone substrates.

By performing steady-state kinetic measurements, we found an inverse correlation of hydrophobicity and velocity. For 1,4-benzoquinone, which is the most water-soluble substrate, the highest turnover number was obtained, whereas duroquinone (whose water solubility is $\sim 50$-fold decreased compared with 1,4-benzoquinone) showed the weakest turnover rate. As the concentration of NADPH is constant for all quinone substrates in Table 1, the observed differences in $k_{\text {cat }}$ values argue that it is quinone reduction, rather than NADPH oxidation, in the first half of the ping-pong mechanism that is rate-limiting in the series of quinone substrates. This assumption is in agreement with the 
kinetic data obtained for human QR type 1; for this enzyme, it was shown that an increased number of rings in the quinone nucleus leads to a decrease in maximal velocity by $88 \%$ [36]. In order to determine which step is rate-determining in the case of Lot6p, future efforts will involve rapid-equilibrium measurements of both half reactions. 1,4-Anthraquinone and coenzyme $\mathrm{Q}_{10}$ were not reduced at all. These results suggest that proper binding of the quinone substrates to the active site is an absolute requirement for catalysis to occur.

Several flavin-dependent oxidoreductases can use quinones as substrates and reduce them to semiquinone radicals in one-electron reactions. Mammalian $\mathrm{NAD}(\mathrm{P}) \mathrm{H}$ :(quinone acceptor) oxidoreductases are a notable exception in that they generate only fully reduced hydroquinones by a compulsory two-electron transfer [38]. Spectral measurements of Lot6p following anaerobic photoreduction exclude the existence of stable flavin semiquinone intermediates. The finding of Liger et al. [14] that Lot6p can also reduce one-electron acceptors such as ferricyanide is in concordance with the present results. Although human QR type 1 also shows activity towards ferricyanide, Tedeschi et al. [39] were unable to detect flavoprotein semiquinones in rapid reaction studies with human QR type 1 and concluded that reduction of the second ferricyanide molecule was much faster than that for the first one. Therefore, a simultaneous two-electron transfer model seems to be appropriate for both human QR type 1 and Lot6p.

Dicoumarol is an efficient inhibitor of quinone reduction by human QR type 1, rat and mouse QR [6]. As has been observed for human QR type 1, inhibition is competitive with respect to $\mathrm{NAD}(\mathrm{P}) \mathrm{H}$ [40]. Hosoda et al. [41] proposed that oxidized QR type 1 possesses an additional dicoumarol binding site and that occupation of this site by dicoumarol results in inhibition of NADPH binding. Recently, Asher et al. [42] reported the crystal structure of human QR type 1 in complex with dicoumarol, clearly indicating that the inhibitor is bound directly to the active site of the enzyme. In the case of Lot6p, our measurements have shown that dicoumarol competitively inhibits quinone reductase activity of Lot6p with respect to NADPH, with an obtained $K_{\mathrm{I}}$ value of $410 \mathrm{~nm}$. The spectral changes that arise upon addition of dicoumarol to Lot6p suggest a comparable binding pattern as observed for human QR type 1.

Similar to dicoumarol, a competitive inhibition pattern was found for Cibacron Marine, an azo dye. This is particularly intriguing since Lot6p was suggested to be an azoreductase due to its sequence similarity to the Bacillus sp. OY1-2 azoreductase [35]. On the other hand, Liger et al. [14] reported a weak reductive activity on azo dyes, i.e. $2-3 \mathrm{nmol}$ of Ethyl Red per minute and milligram of protein, which is several orders of magnitude lower than the activity reported for bacterial azoreductases.

In addition to the biochemical characterization of Lot6p as a quinone reductase, we were also interested in whether this in vitro activity can be regarded as the physiological role of this enzyme. The metabolism of quinones within a cell has a direct effect on the cell's ability to cope with oxidative stress [43]. Quinone compounds can be reduced by certain oxidoreductases via a one-electron transfer to give semiquinones, which in turn are able to react with molecular oxygen to form superoxide radicals. In contrast, cytosolic NAD $(\mathrm{P}) \mathrm{H}$ : quinone oxidoreductases catalyze the two-electronreduction of quinones to quinols, therefore competing with the potentially toxic one-electron pathway and protecting against the cytotoxic effect of quinones. In order to determine whether this protective function can be attributed to Lot6p in yeast, we assayed the oxidative stress resistance of wild type, Alot6 and Lot6p overexpressing strains by testing the ability of 1,4-naphthoquinone to reduce their growth rate. Our results demonstrate that the enzymatic activity of Lot6p is consistent with the phenotype of both $\Delta$ lot 6 and Lot6p overexpressing strains and that expression of Lot6p is undoubtedly important for managing oxidative stress in yeast. In this context, it is important to note that the cytosolic localization of Lot6p was confirmed by two independent methods, pointing towards a functional role in this compartment. Such a role is also highlighted by the recent finding that human QR type 1 is specifically associated with the $20 \mathrm{~S}$ proteasome [44]. It will be interesting to see whether this proteinprotein interaction is conserved in yeast and which role it plays in yeast physiology.

\section{Experimental procedures}

\section{Reagents}

All chemicals were of highest grade commercially available and purchased from Sigma-Aldrich (St Louis, MO, USA), Fluka (Buchs, Switzerland) or Merck (Darmstadt, Germany). Ni-NTA agarose was obtained from Qiagen (Hilden, Germany) and HiTrap Blue HP column from Amersham Biosciences (Uppsala, Sweden).

\section{Strains}

The $S$. cerevisiae yeast strains Y00000 (BY4741 Mata his341

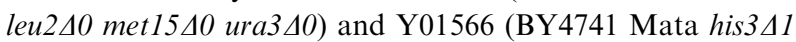


leu2 40 met15 40 ura3 $\Delta 0$ lot $6 \Delta: \because K a n M X 4)$ used in this study were obtained from the Euroscarf strain collection [16]. Strains were grown under aerobic conditions at $30{ }^{\circ} \mathrm{C}$ on complete yeast-peptone-dextrose medium containing $1 \%$ yeast extract, $2 \%$ peptone, and $2 \%$ glucose as the carbon source or on synthetic minimal medium containing $2 \%$ glucose $(\mathrm{SD})$ or $2 \%$ galactose $(\mathrm{SG}), 0.67 \%$ yeast nitrogen base without amino acids and with ammonium sulfate, supplemented with the appropriate amino acids. DNA cloning and propagation of plasmids was performed in E. coli Strain TOP10 (Invitrogen, Carlsbad, CA, USA). For Expression of Lot6p-His6, E. coli Rosetta(DE3)pLysS (Novagen, Darmstadt, Germany) was used.

\section{Preparation of subcellular fractions and protein analysis}

For the isolation of mitochondria, microsomes, nuclei and vacuoles, late exponential phase cultures were harvested by centrifugation and converted to spheroplasts essentially as described by Daum et al. [17]. Mitochondria were isolated as describe previously [17]. Microsomal membranes were obtained from the postmitochondrial supernatant by differential centrifugation at $40000 \mathrm{~g}$ and $100000 \mathrm{~g}$; the $100000 \mathrm{~g}$ supernatant being the soluble cytosolic fraction $[17,18]$. Nuclei were enriched by sucrose density gradient centrifugation of the postmitochondrial supernatant as described $[19,20]$. Vacuoles were isolated as described by Uchida et al. [21]. Plasma membranes were isolated from cell homogenates obtained by glass beading following the procedure described by Serrano [22]. Relative enrichment of markers and cross-contamination of subcellular fractions were assessed as described by Zinser and Daum [23]. Protein was quantified by the method of Lowry et al. [24] by using bovine serum albumin as a standard. SDS/PAGE was carried out as published by Laemmli [25]. Western blot analysis of proteins from subcellular fractions prepared as described above was performed as described by Haid and Suissa [26] using a primary rabbit antibody against Lot6p. The antibody against Lot6p (anti-Lot6p) was raised in rabbits by immunization with recombinant Lot6p (see below) according to standard procedures. Immunoreactive bands were visualized by enzyme-linked immunosorbent assay using a peroxidase-linked secondary antibody (SigmaAldrich) following the manufacturer's instructions.

\section{Microscopic inspection}

Strains expressing GFP-Lot6p were grown to the logarithmic growth phase on SG medium and put onto microscope slides. Fluorescence and light microscopy was performed using a Zeiss Axiovert 35 microscope with a 100-fold oil immersion objective and a UV lamp with the appropriate filters for fluorescence detection. The detection range of $510-520 \mathrm{~nm}$ was used for green fluorescent protein (GFP) inspection. Cells were also labeled with the nucleic acid stain DAPI (Molecular Probes, Carlsbad, CA, USA) according to the manufacturer's instructions. Images were taken with a charge-coupled device camera.

\section{Construction of GFP-Lot6p fusions}

Genomic N-terminal GFP fusions of LOT6 were constructed by insertion of a His3MX6-PGAL1-GFP cassette upstream of the START codon of the LOT6 ORF. Primers LOT6-F4 (5'-CATTACCAGCTGAGGTGCTTAGTCGA CAACCGCATTAAACGAATTC-GAGCTCGTTTAAAC -3') and LOT6-R5 (5'- CCCTCTTTGCCCTCACAGAA CCCATTA-TAATACCCACTTTTTTGTATAGTTCATC CATGC-3') were used to amplify the cassette from plasmid pFA6a-His3MX6-PGAL1-GFP by polymerase chain reaction [27]. The cassette was introduced into strain Y00000 by lithium acetate transformation [28]. Correct insertion of the cassette was tested by growth of strains on selective media without the respective amino acid and by colony PCR with the appropriate primers.

\section{Cloning of LOT6 for large scale expression in $E$. coli and for yeast overexpression}

A plasmid containing the LOT6 gene of S. cerevisiae was a generous gift from Prof Herman van Tilbeurgh, Université Paris-Sud, France. The gene was amplified by the PCR from this plasmid DNA, using the following primers: 5'-GGAATTCCATATGAAAGTGGGTATTATAATGG; 3'-CCGCTCGAGTTTATTCCTCGTTGTTTCG and inserted into the $N d e \mathrm{I} / \mathrm{XhoI}$ restriction sites of pET21a (Novagen) to create the expression construct pET21a-LOT6HIS. Cloning into this vector using $N d e \mathrm{I} / \mathrm{XhoI}$ and deletion of the stop codon allows expression of the protein with a hexa-histidine affinity tag at the C-terminus. For cloning LOT6 into the yeast shuttle vector pYES2 (Invitrogen) the following primers were used: 5'-CCCAAGCTTGGGACCA TGAAAGTGGGTATTATAATGG; 3'-CCGCTCGAGCG GTTATTTATTCCTCGTTGTTTCG: The PCR product was digested with HindIII and XhoI and ligated into the corresponding sites of the vector pYes2, thus expressing Lot6p under the control of the promotor Gall.

\section{Expression and purification of $S$. cerevisiae Lot6p-His6}

A single colony of E. coli Rosetta(DE3)pLysS harboring pET21a-LOT6HIS was grown overnight in $5 \mathrm{~mL}$ of Luria broth supplemented with $100 \mu \mathrm{g} \cdot \mathrm{mL}^{-1}$ ampicillin and $36 \mu \mathrm{g} \cdot \mathrm{mL}^{-1}$ chloramphenicol, which was then used to inoculate a $500 \mathrm{~mL}$ culture. After $3 \mathrm{~h}$ at $37^{\circ} \mathrm{C}$, expression was induced by the addition of isopropyl-1-thio-D-galactopyranoside to a final concentration of $0.5 \mathrm{~mm}$. Cells were allowed to 
grow for another $3 \mathrm{~h}$ at $37^{\circ} \mathrm{C}$ and then harvested by centrifugation and subsequently stored at $-70{ }^{\circ} \mathrm{C}$. For protein purification, $4 \mathrm{~L}$ of $E$. coli cells expressing Lot6p-His6 were grown in parallel and the resulting bacterial paste was resuspended in $50 \mathrm{~mm}$ sodium phosphate at $\mathrm{pH} 8.0$ containing $300 \mathrm{~mm}$ sodium chloride and $10 \mathrm{~mm}$ imidazole (buffer $\mathrm{A}$ ) as the resuspension buffer. The cells were lysed by five cycles of sonication, $1 \mathrm{~min}$ each. Cell debris were removed by centrifugation at $25000 \mathrm{~g}$ for $20 \mathrm{~min}$ at $4{ }^{\circ} \mathrm{C}$, and the supernatant was subjected to affinity chromatography on Ni-NTA resin, essentially according to protocol number 11 provided by the supplier (Qiagen). In particular, buffer A containing $20 \mathrm{~mm}$ imidazole was used to wash the column and the bound protein was eluted in buffer A containing $150 \mathrm{~mm}$ imidazole. The progress of Lot6p-His6 purification was monitored by $12.5 \%$ SDS/PAGE [25].

The concentration of the Lot6p-His6 preparation was determined spectrophotometrically at $455 \mathrm{~nm}$ using a molar extinction coefficient of $10690 \mathrm{M}^{-1} \cdot \mathrm{cm}^{-1}$, which was calculated by recording differential spectra of the native and denatured protein, using the known extinction coefficient for authentic FMN of $\varepsilon_{450}=12500 \mathrm{M}^{-1} \cdot \mathrm{cm}^{-1}$.

All fractions of high purity and an appropriate amount of enzyme (according to the SDS/PAGE) were pooled and concentrated by centrifugation with the CentriPrep System from Amicon (molecular mass cut-off of $10 \mathrm{kDa}$ ). This system required repeated centrifugation steps at $1700 \mathrm{~g}$ for $20 \mathrm{~min}$ at $4{ }^{\circ} \mathrm{C}$ (Hereaus Multifuge $3 \mathrm{~S}-\mathrm{R}$ ) until the final buffer $(50 \mathrm{~mm}$ phosphate at $\mathrm{pH} 8.0)$ was added. Aliquots of $1 \mathrm{~mL}$ were stored at $4{ }^{\circ} \mathrm{C}$.

\section{Influence of Lot6p on quinone toxicity}

Quinone toxicity of yeast strains was analyzed as described by Rodriguez et al. [29]. A single colony of strain Y00000 transformed with pYes2-LOT6 or empty plasmid pYes2 and Y01566 harboring pYes2 (herein after referred as 'Lot6p overexpression strain', 'wild-type strain' and ' $\Delta$ lot6 strain', respectively) was grown overnight at $30^{\circ} \mathrm{C}$ in SG-uracil. Precultures were then diluted to a final optical density $\left(\mathrm{OD}_{600}\right)$ of $0.25-0.3$. After addition of $10 \mu \mathrm{M}$ 1,4-naphthoquinone in dimethyl sulfoxide growth at $30^{\circ} \mathrm{C}$ was photometrically monitored at $1-\mathrm{h}$ intervals for $8 \mathrm{~h}$. The final dimethyl sulfoxide concentration was $0.1 \%$ in the culture containing quinone, as well as in the control culture. The results are expressed as the percentage of viability relative to the untreated culture.

\section{Steady-state kinetic studies}

Reactions were conducted in $1.0 \mathrm{~mL}$ of $50 \mathrm{~mm}$ sodium phosphate, $\mathrm{pH} 8.0$, with $5 \mathrm{nM}$ of enzyme at $37^{\circ} \mathrm{C}$. Quinones were dissolved in neat ethanol. The low aqueous solubility of the quinones studied and the inactivation of the enzyme at higher ethanol concentrations limited the maximal concentration of quinone substrates to $100 \mu \mathrm{M}$. NADPH concentrations ranged from 75 to $200 \mu \mathrm{M}$. The reactions were initiated by the addition of NADPH. Initial velocities were measured by monitoring the decrease in absorbance at $340 \mathrm{~nm}\left(\varepsilon_{340}=\right.$ $6220 \mathrm{M}^{-1} \cdot \mathrm{cm}^{-1}$ ) owing to NADPH oxidation. Control reactions were performed in the absence of enzyme.

\section{Spectrophotometric methods}

Lot6p-bound FMN was released by addition of $0.1 \%$ SDS. UV-visible absorbance spectra were recorded before and after denaturation of the enzyme.

\section{Photoreduction}

Lot6p was photoreduced anaerobically using a 50-W halogen lamp as a light source. Anaerobic conditions were established by repeated cycles of evacuation and flushing with oxygen-free nitrogen gas. Solutions contained $60 \mu \mathrm{M}$ Lot6p in $100 \mathrm{~mm}$ Tris/ $\mathrm{HCl}$ buffer, $\mathrm{pH}$ 8.0.

\section{Determination of redox potential}

The redox potential for Lot6p $(30 \mu \mathrm{M})$ was determined in $50 \mathrm{~mm}$ phosphate buffer, $\mathrm{pH} 7.0,25.0{ }^{\circ} \mathrm{C}$, containing about $25 \mu \mathrm{M}$ 1-hydroxyphenazine as a redox dye and $2 \mu \mathrm{M}$ methyl viologen as a mediator by titration with dithionite ( $1 \mathrm{mg} \cdot \mathrm{mL}^{-1}$ in $50 \mathrm{~mm}$ phosphate, $\left.\mathrm{pH} 7.0\right)$ under anaerobic conditions [30].

\section{Inhibition of Lot6p activity by dicoumarol and Cibacron Marine}

The initial rate of NADPH oxidation was determined in the presence of various concentrations $(0-4 \mu \mathrm{M})$ of dicoumarol and Cibacron Marine. Reaction mixtures $(1 \mathrm{~mL})$ contained $50 \mathrm{~mm}$ sodium phosphate, $\mathrm{pH} 8.0, \quad 10 \mu \mathrm{M}$ 1,4-benzoquinone, $\quad 50-150 \mu \mathrm{M}$ NADPH and enzyme $(10 \mathrm{nM})$. The reactions were initiated by addition of NADPH. A stock solution of $10 \mathrm{~mm}$ dicoumarol was prepared in $0.1 \mathrm{M} \mathrm{NaOH}$. Cibacron Marine $(200 \mu \mathrm{M})$ was prepared in $\mathrm{H}_{2} \mathrm{O}$.

\section{Titration of apoprotein with FMN}

Apoenzyme was prepared using a HiTrap Blue affinity column $(5 \mathrm{~mL})$ by Amersham. Up to $10 \mathrm{mg}$ of Lot6p were applied to the column after equilibration with $30 \mathrm{~mL}$ of phosphate buffer (50 mM, pH 8.0). The column was washed with $30 \mathrm{~mL}$ of the same buffer to elute the flavin cofactor. Finally, bound apoenzyme was eluted using the same buffer containing $1.5 \mathrm{M} \mathrm{KCl}$. The pooled protein-containing fractions were dialyzed and concentrated as described above. Holoenzyme was reconstituted from the apoenzyme at 
$10{ }^{\circ} \mathrm{C}$ by the stepwise addition of $1-2 \mu \mathrm{mol}$ aliquots of FMN. After each addition of FMN, the sample was incubated for $2 \mathrm{~min}$ at $10{ }^{\circ} \mathrm{C}$.

\section{Acknowledgements}

This work was supported in part by the Fonds zur wissenschaftlichen Forschung (FWF) through the PhD program 'Molecular Enzymology' (W901-B05 DK Molecular Enzymology to P. Macheroux) and by NIH Grant GM61087 to B. A. Palfey. The authors would like to thank Professor $H$. van Tilbeurgh from the University of Paris XI, Orsay, France for the generous gift of both a plasmid carrying the coding sequence of LOT6 and the expression strain.

\section{References}

1 Ernster L (1987) DT-diaphorase - a historical review. Chemica Scripta 27A, 1-13.

2 Radjendirane V, Joseph P, Lee Y-H, Kimura S, KleinSzanto AJP, Gonzalez FJ \& Jaiswal AK (1998) Disruption of the DT diaphorase (NQO1) gene in mice leads to increased menadione toxicity. J Biol Chem 273, 7382-7389.

3 Ross D, Kepa JK, Winski SL, Beall HD, Anwar A \& Siegel D (2000) NAD(P)H: quinone oxidoreductase 1 (NQO1): Chemoprotection, bioactivation, gene regulation and genetic polymorphisms. Chem-Biol Interact 129, 77-97.

4 Faig M, Bianchet MA, Talalay P, Chen S, Winski S, Ross D \& Amzel LM (2000) Structures of recombinant human and mouse NAD(P)H: quinone oxidoreductases: species comparison and structural changes with substrate binding and release. Proc Natl Acad Sci USA 97, 3177-3182.

5 Foster CE, Bianchet MA, Talalay P, Zhao Q \& Amzel LM (1999) Crystal structure of human quinone reductase type 2, a metalloflavoprotein. Biochemistry 38, 9881-9886.

6 Li R, Bianchet MA, Talalay P \& Amzel LM (1995) The three-dimensional structure of $\mathrm{NAD}(\mathrm{P}) \mathrm{H}$ : quinone reductase, a flavoprotein involved in cancer chemoprotection and chemotherapy: mechanism of the two-electron reduction. Proc Natl Acad Sci USA 92, 8846-8850.

7 Thorn JM, Barton JD, Dixon NE, Ollis DL \& Edwards KJ (1995) Crystal structure of Escherichia coli QOR quinone oxidoreductase complexed with NADPH. J Mol Biol 249, 785-799.

8 Brock BJ \& Gold MH (1996) 1,4-Benzoquinone reductase from the Basidiomycete Phanerochaete chrysosporium: spectral and kinetic analysis. Arch Biochem Biophys 331, 31-40.
9 Prochaska HJ, De Long MJ \& Talalay P (1985) On the mechanisms of induction of cancer-protective enzymes: a unifying proposal. Proc Natl Acad Sci USA 82, 82328236.

10 Prochaska HJ \& Talalay P (1991) In Oxidative Stress: Oxidants and Antioxidants (Sies, H, eds), pp. 195-211. Academic Press, London.

11 Favreau LV \& Pickett CB (1991) Transcriptional regulation of the rat $\mathrm{NAD}(\mathrm{P}) \mathrm{H}$ : quinone reductase gene: identification of regulatory elements controlling basal level expression and inducible expression by planar aromatic compounds and phenolic antioxidants. $J$ Biol Chem 266, 4556-4561.

12 Denison MS \& Fisher JM (1988) The DNA recognition site for the dioxin-Ah receptor complex: nucleotide sequence and functional analysis. $\mathrm{J}$ Biol Chem 263, 17221-17224.

13 Zhang L, Ohta A, Horiuchi H, Takagi M \& Imai R (2001) Multiple mechanisms regulate expression of low temperature responsive (LOT) genes in Saccharomyces cerevisiae. Biochem Biophys Res Commun 283, 531-535.

14 Liger D, Graille M, Zhou CZ, Leulliot N, QuevillonCheruel S, Blondeau K, Janin J \& van Tilbeurgh H (2004) Crystal structure and functional characterization of yeast YLR011wp, an enzyme with NAD(P)H-FMN and ferric iron reductase activities. J Biol Chem 279, 34890-34897.

15 Deller S, Sollner S, Trenker-El-Toukhy R, Jelesarov I, Gübitz GM \& Macheroux P (2006) Characterization of a thermostable NADPH: FMN oxidoreductase from the mesophilic bacterium Bacillus subtilis. Biochemistry 45, 7083-7091.

16 Winzeler EA, Shoemaker DD, Astromoff A, Liang H, Anderson K, Andre B, Bangham R, Benito R, Boeke JD, Bussey H, et al. (1999) Functional characterization of the S. cerevisiae genome by gene deletion and parallel analysis. Science 285, 901-906.

17 Daum G, Böhni PC \& Schatz G (1982) Import of proteins into mitochondria: cytochrome b2 and cytochrome c peroxidase are located in the intermembrane space of yeast mitochondria, J Biol Chem 257, 13028-13033.

18 Zinser E, Sperka-Gottlieb CDM, Fasch E-V, Kohlwein SD, Paltauf F \& Daum G (1991) Phospholipid synthesis and lipid composition of subcellular membranes in the unicellular eukaryote Saccharomyces cerevisiae. $J$ Bacteriol 173, 2026-2034.

19 Hurt EC, McDowall A \& Schimmang T (1988) Nucleolar and nuclear envelope proteins of the yeast Saccharomyces cerevisiae. Eur J Cell Biol 46, 554-563.

20 Aris JP \& Blobel G (1991) Isolation of yeast nuclei. Methods Enzymol 194, 735-749.

21 Uchida E, Ohsumi Y \& Anraku Y (1988) Purification of yeast vacuolar membrane $\mathrm{H}^{+}$-ATPase and enzymological discrimination of three ATP-driven proton 
pumps in Saccharomyces cerevisiae. Methods Enzymol 157, 544-562.

22 Serrano R (1988) $\mathrm{H}^{+}$-ATPase from plasma membranes of Saccharomyces cerevisiae and Avena sativa roots: purification and reconstitution. Methods Enzymol 157, 523-544

23 Zinser E \& Daum G (1995) Isolation and biochemical characterization of organelles from the yeast Saccharomyces cerevisiae. Yeast 11, 493-536.

24 Lowry OH, Rosebrough NJ, Farr AL \& Randall RJ (1951) Protein measurement with the folin phenol reagent. J Biol Chem 193, 265-275.

25 Laemmli UK (1970) Cleavage of structural proteins during the assembly of the head of bacteriophage T4. Nature 227, 680-685.

26 Haid A \& Suissa M (1983) Immunochemical identification of membrane proteins after sodium dodecyl sulfatepolyacrylamide gel electrophoresis. Methods Enzymol 96, 192-205.

27 Longtine MS, McKenzie A, Demarini DJ, Shah NG, Wach A, Brachat A, Philippsen P \& Pringle JR (1998) Additional modules for versatile and economical PCRbased gene deletion and modification in Saccharomyces cerervisiae. Yeast 14, 953-961.

28 Gietz D, St. Jean A, Woods RA \& Schiestl RH (1992) Improved method for high efficiency transformation of intact yeast cells. Nucleic Acids Res 20, 1425.

29 Rodriguez CE, Shinyashiki M, Froines JYuRC, Fukuto JM \& Cho AK (2004) An examination of quinone toxicity using the yeast Saccharomyces cerevisiae model system. Toxicology 201, 185-196.

30 Massey V (1990) Flavin and Flavoproteins (Curti, B, Ronchi, S, \& \& Zanetti, G, eds), pp. 59-66. Walter de Gruyter \& Co, Berlin.

31 Kumar A, Agarwal S, Heyman JA, Matson S, Heidtman M, Piccirillo S, Umansky L, Drawid A, Jansen R, Liu Y, et al. (2002) Subcellular localization of the yeast proteome. Genes Dev 16, 707-719.

32 Huh W-K, Falvo JV, Gerke LC, Carroll AS, Howson RW, Weissman JS \& O'Shea EK (2003) Global analysis of protein localization in budding yeast. Nature 425, 686-691.

33 Müller O (1942) Oxidation-reduction potentials measured with the dropping mercury electrode. $J$ Biol Chem 145, 425-441.
34 Minnaert K (1965) Measurement of the equilibrium constant of the reaction between cytochrome $\mathrm{c}$ and cytochrome a. Biochim Biophys Acta 110, 42-56.

35 Suzuki Y, Yoda T, Ruhul A \& Sugiura W (2001) Molecular cloning and characterization of the gene coding for azoreductase from Bacillus sp. OY1-2 isolated from soil. J Biol Chem 276, 9059-9065.

36 Trost P, Bonora P, Scagliarini S \& Pupillo P (1995) Purification and properties of NAD $(\mathrm{P}) \mathrm{H}$ : (quinoneacceptor) oxidoreductase of sugarbeet cells. Eur J Biochem 234, 452-458.

37 Zhou Z, Fisher D, Spidel J, Greenfield J, Patson B, Fazal A, Wigal C, Moe OA \& Madura JD (2003) Kinetic and docking studies of the interaction of quinones with the quinone reductase active site. Biochemistry 42, 1985-1994.

38 Iyanagi T (1987) On the mechanisms of one- and twoelectron transfer by flavin enzymes. Chemica Scripta 27A, 31-36.

39 Tedeschi G, Chen S \& Massey V (1995) DT-diaphorase: redox potential, steady-state, and rapid reaction studies. J Biol Chem 270, 1198-1204.

40 Prestera T, Prochaska HJ \& Talalay P (1992) Inhibition of NAD(P)H: (quinone-acceptor) oxidoreductase by cibacron blue and related anthraquinone dyes: a structure-activity study. Biochemistry 31, 824-833.

41 Hosoda S, Nakamura W \& Hayashi K (1974) Properties and reaction mechanism of DT diaphorase from rat liver. J Biol Chem 249, 6416-6423.

42 Asher G, Dym O, Tsvetkov P, Adler J \& Shaul Y (2006) The crystal structure of NAD(P)H quinone oxidoreductase 1 in complex with its potent inhibitor dicoumarol. Biochemistry 45, 6372-6378.

43 Soballe B \& Poole RK (1999) Microbial ubiquinones: multiple roles in respiration, gene regulation and oxidative stress management. Microbiology 145, 1817-1830.

44 Asher G, Tsvetkov P, Kahana C \& Shaul Y (2005) A mechanism of ubiquitin-independent proteasomal degradation of the tumor suppressors p53 and p73. Genes Dev 19, 316-321.

45 Volland C, Urban-Grimal D, Geraud G \& HaguenauerTsapis R (1994) Endocytosis and degradation of the yeast uracil permease under adverse conditions. $J$ Biol Chem 269, 9833-9841. 